\title{
BMJ Open Impact of sex work on risk behaviours and their association with HIV positivity among people who inject drugs in Eastern Central Canada: cross- sectional results from an open cohort study
}

\author{
Laurence Campeau, ${ }^{1,2}$ Karine Blouin, ${ }^{1}$ Pascale Leclerc, ${ }^{2,3}$ Michel Alary, ${ }^{1,4,5}$ \\ Carole Morissette, ${ }^{3}$ Caty Blanchette, ${ }^{4}$ Bouchra Serhir, ${ }^{6}$ Elise Roy, ${ }^{1,7}$ on behalf of the
} SurvUDI Working Group

To cite: Campeau L, Blouin K, Leclerc P, et al. Impact of sex work on risk behaviours and their association with HIV positivity among people who inject drugs in Eastern Central Canada: crosssectional results from an open cohort study. BMJ Open 2018;8:e019388. doi:10.1136/ bmjopen-2017-019388

- Prepublication history for this paper is available online. To view these files, please visit the journal online (http://dx.doi org/10.1136/bmjopen-2017019388).

Received 31 August 2017 Revised 22 November 2017 Accepted 19 December 2017

Check for updates

For numbered affiliations see end of article.

Correspondence to Dr Karine Blouin; karine.blouin@inspq.qc.ca

\section{ABSTRACT}

Objectives The objectives of this study were: (1) to examine the correlates of HIV positivity among participants who injected drugs and engaged in sex work (PWID-SWs) in the SurvUDI network between 2004 and 2016, after stratification by sex, and (2) to compare these correlates with those of sexually active participants who did not engage in sex work (PWID non-SWs).

Design and setting This biobehavioural survey is an open cohort of services where participants who had injected in the past 6 months were recruited mainly through harm reduction programmes in Eastern Central Canada. Participants Data from 5476 participants (9223 visits in total; 785 not included in multivariate analyses due to missing values) were included.

Methods Participants completed an intervieweradministered questionnaire and provided saliva samples for anti-HIV antibody testing. Generalised estimating equations taking into account multiple participations were used.

Results Baseline HIV prevalence was higher among SWs compared with non-SWs (women: $13.0 \%$ vs $7.7 \%$; $\mathrm{P}<0.001$, and men: $17.4 \%$ vs $10.8 \% ; \mathrm{P}<0.001$ ). PWIDSWs were particularly susceptible to HIV infection as a result of higher levels of vulnerability factors and injection risk behaviours. They also presented different risk-taking patterns than their non-SWs counterparts, as shown by differences in correlates of HIV positivity. Additionally, the importance of sex work for HIV infection varies according to gender, as suggested by a large proportion of injection risk behaviours associated with HIV among women and, conversely, a stronger association between sexual behaviours and HIV positivity observed among men. Conclusion These results suggest that sex work has an impact on the risk of HIV acquisition and that risk behaviours vary according to gender. Public health practitioners should take those specificities into account when designing HIV prevention interventions aimed at PWIDs.
Strengths and limitations of this study

- The study used a large geographical coverage including eight Quebec regions and Ottawa as well as urban and rural sites.

- The method was stable over time and the questionnaire was comprehensive, including injection behaviours, sexual behaviours and some clinical informations.

- Participants might not be representative of all participants who injected drugs in Eastern Central Canada since they were mostly recruited through harm reduction programmes.

- Similarly to other observational cohort studies, social desirability and recall biases cannot be ruled out due to the use of self-reported measures.

- Causal relationships could not be established owing to the use of prevalent cases. Behaviours may have occurred before or after the time of infection.

\section{INTRODUCTION}

People who inject drugs (PWIDs) are a highly marginalised segment of the population as they often experience a variety of challenges, including stigmatisation, discrimination, addiction, economic pressure and social exclusion. Those factors are often associated with high-risk income-generating activities, including the exchange of sex for money, drugs, goods or other things, resulting in an overlap between drug injection and sex work. ${ }^{1}$

PWIDs who also engage in sex work (PWID-SWs) have been identified as a key group with respect to HIV infection. In addition to the impact of being exposed to both injection-related and sexual transmission 
pathways, ${ }^{2}$ the criminalisation and stigmatisation of both drug use and sex trade in most countries might lead to an increased risk of health harms, including blood-borne viruses (BBVs). ${ }^{3}$ Additionally, people who engage in both sex work and drug injection require special attention due to potentially important public health consequences. Given that the HIV epidemic in North America is known to be concentrated among specific key populations, they can potentially serve as a bridge between those and lower risk populations. ${ }^{4}$

Studies conducted in the European region have shown a high risk of HIV infection among people who inject drugs and sell sex. ${ }^{5-7}$ Nonetheless, with limited HIV/ AIDS surveillance data among sex workers (SWs) in North American settings, few studies have examined in detail the association between HIV infection, injection drug use and sex trade involvement in this region. ${ }^{18} \mathrm{~A}$ study published in 2011 highlighted the need for more evidence on this topic by documenting the emergence of sex work as an independent risk factor for HIV infection among PWIDs in Eastern Central Canada. ${ }^{9}$ A subsequent study showed that HIV incidence among PWID-SWs was 2.19 times higher than among those not reporting client sex partners for the 2004-2014 period (adjusted HR: $2.19,95 \%$ CI 1.13 to 4.25$).{ }^{10}$ The risk factors for HIV incidence among this key population were also explored, but limited number of seroconversions resulted in a small amount of variables being studied. ${ }^{10}$

Thus, we undertook the present analyses to identify the correlates of HIV positivity among participants who injected and engaged in sex work, after stratification according to sex, in the SurvUDI network, an ongoing biobehavioural survey among PWIDs in Eastern Central Canada. We also compared these correlates with those of sexually active participants who did not engage in sex work. We hypothesised that, in addition to injection behaviours, risky sexual behaviours would be associated with HIV positivity, especially in men reporting sex work and sex with men.

\section{METHODS}

\section{Study design and subjects}

The complete methodology of the SurvUDI study has been described elsewhere. ${ }^{11}$ Briefly, the SurvUDI network is an ongoing biobehavioural survey for HIV, hepatitis $\mathrm{C}$ virus (HCV) and associated risk behaviours among PWIDs in Eastern Central Canada. The network was implemented in 1995 and targets hard-to-reach, mostly out-of-treatment PWIDs. Eligibility criteria include being aged $\geq 14$ years, injecting at least once within the past 6 months, speaking French or English and being able to provide informed consent. Participants are recruited in urban areas, including Montréal and neighbouring South Shore, Québec City, the Hull-Ottawa region and five semiurban areas of the province of Québec. Overall, since 2004, 95.2\% of participants were recruited in harm reduction programmes. Others were recruited in drop-in centres, detention centres, detoxification clinics and rehabilitation programmes. Participation includes an interviewer-administered questionnaire and collection of gingival exudate using the Orasure device (Bethlehem, Pennsylvania, USA) for HIV and HCV antibody testing. The study design is an open cohort of services where participants who attend harm reduction programme are recruited. PWIDs may participate more than once and be followed longitudinally. The present sample includes sexually active participants recruited from 1 March 2004 to 31 March 2016. Participants are identified using an encrypted code based on their initials, birth date and sex, and they are given a stipend ranging from CAN\$5.00 to $\$ 10.00$ at the end of each study visit.

\section{Study variables}

The dependent variable was HIV positivity. Potential correlates of HIV positivity were identified based on a literature review and on previous analyses on this cohort. ${ }^{10}{ }^{12}$ Variables considered in univariate analyses included age, high school not completed, homelessness, recent incarceration, the region of recruitment (urban or semiurban/rural), year of recruitment, time since first injection ( $\geq 6$ years), injection partners (always injecting alone, mainly with known people, mainly with strangers), injection with a syringe used by someone else and injection with material other than syringes, such as mixing containers, filters and cottons, used by someone else (never, mainly obtained from known people, mainly obtained from strangers), not having lent their used syringes to others, daily injection in the last month, high number of injections in the last month $(\geq 120)$, cocaine as the most often injected drug, crack/freebase use other than by injection, injection of two drugs or more, sex of sexual partners (only men, only women, both men and women), sexual intercourse in the last month, having at least one regular sex partner, having at least one casual sex partner, having anal, vaginal or oral sex according to partner type (regular, casual and client), high number of male sex partners ( $\geq 21$ partners), consistent condom use for vaginal and anal sex (always, not always, no anal or vaginal intercourse; and separately for anal, vaginal and oral sex according to partner type, ie, regular, casual and client), and condom use at the last sexual intercourse. Sex work was defined as having client sex partners in the past 6 months, that is, partners giving money, drugs, goods or other things in exchange for sex. Unless otherwise stated, questions about behaviours referred to the 6 months prior to the interview.

\section{Laboratory procedures}

Collected oral fluid samples were kept at $4^{\circ} \mathrm{C}$ and shipped within 2 weeks to the Laboratoire de santé publique du Québec (Institut national de santé publique du Québec), where they were centrifuged on reception. The extracted liquid was kept at $-20^{\circ} \mathrm{C}$ for a maximum of 6 weeks until analysis. The presence of HIV antibodies was assessed by enzyme immunoassay (EIA) using HIV-1 Vironostika 
Microelisa System (bioMérieux, Durham, North Carolina, USA) from 2004 to 2009 and GS HIV-1/HIV-2 PLUS O EIA (Bio-Rad Laboratories (Canada) Ltd, Montréal, Qc, Canada) thereafter. The presence of HCV antibodies was assessed using ORTHO HCV 3.0 ELISA Test System (BioRad Laboratories (Canada) Ltd, Montréal, Qc, Canada) according to a modified method developed by Judd $e t \mathrm{al}^{13}$ Samples were considered negative if results were less than $75 \%$ of the cut-off value. Sample results that were greater than $75 \%$ of the cut-off value were retested in duplicate. A sample was deemed positive if at least two out of three results were greater than the cut-off value.

\section{Statistical analyses}

Cross-sectional sex-stratified descriptive analyses were conducted to compare risk profiles at baseline between participants engaged in SW and other participants. Pearson's $\mathrm{X}^{2}$ tests were used for categorical variables and two-sample t-test were used for continuous variables, with Satterthwaite's correction when variances were unequal.

Univariate and multivariate generalised estimating equations with Poisson regression and robust variance were carried out for each group (SWs and non-SWs, analysed separately by sex) to assess correlates of HIV positivity, ${ }^{14}{ }^{15}$ with the prevalence ratio (PR) used as the measure of association. Data collected at all visits were used, and 785 visits were not included in multivariate analyses due to missing values. Variables were considered for inclusion in the multivariate analyses if they had a $P$ value of $\leq 0.20$ in the univariate analysis. The final multivariate analyses included significant variables $(\mathrm{P}<0.05)$ and confounders, that is, variables changing PRs by $>10 \%$ when removed from the complete model. Variables that were significant or confounders in one or the other of the two groups (SWs or non-SWs), by sex, were included in both analyses. All analyses were conducted with the SAS statistical suite software V.9.4 (SAS Institute, Cary, North Carolina, USA).

\section{RESULTS}

\section{Characteristics of participants at baseline}

A total of 5476 sexually active PWIDs recruited between 2004 and 2016 were included in our analyses. Women comprised $28 \%$ of the sample, and respectively $34 \%$ (517) and 7\% (286) of female and male participants reported sex work. Among the HIV-positive participants, including both sexes as well as SWs and non-SWs, 78.7\% were aware of their status. Among those, $87.9 \%$ had consulted a doctor about HIV in the past 6 months, and $59.3 \%$ were on antiretroviral therapy (results not shown). Baseline characteristics of the participants are shown in table 1.

\section{Women}

Overall, the mean age among female participants was 32 years. HIV and HCV prevalence at baseline were higher among female SWs compared with non-SWs (HIV: 13.0\% vs 7.7 , HCV: $70.4 \%$ vs $53.7 \%$ ). While recruitment region and high school completion were similar among both groups, a higher proportion of female SWs had been incarcerated and reported being homeless in the past 6 months.

Among female SWs, $11 \%$ and $10 \%$, respectively, reported injection with a syringe and other material used by someone else that had mainly been obtained from strangers, compared with $3 \%$ and $4 \%$ among non-SWs. Injecting mainly with strangers and reporting having lent used syringes was also more frequent among SWs. Duration and frequency of injection differed by SW status, with female SWs being more likely than non-SWs to have been injecting for at least 6 years and to report at least 120 injections in the past month. A higher proportion of female SWs also reported cocaine as the most often injected drug as well as the consumption of crack/freebase other than by injection.

Conversely, 32\% of female SWs reported the consistent use of condoms for vaginal and anal intercourse, as opposed to $18 \%$ among non-SWs. SWs were also more likely to have used condoms at their last sexual intercourse and to report $>21$ male sexual partners.

\section{Men}

The mean age among male participants was 36 years. Baseline HIV prevalence was higher among male SWs compared with non-SWs $(17.4 \%$ vs $10.8 \%)$, but this was not the case for HCV prevalence. A higher proportion of male SWs reported having been homeless in the past 6 months in comparison with non-SWs.

Respectively $20 \%$ and $16 \%$ of male SWs reported injection with a syringe and other material used by someone else mainly obtained from strangers, compared with $5 \%$ and $6 \%$ among non-SWs. Injecting mainly with strangers and having lent used syringes to others was also more frequent among SWs. Proportionally more male SWs reported having injected at least 120 times in the past month, reported cocaine as their most often injected drug, and used crack/freebase other than by injection compared with non-SWs.

Male SWs were less likely to consistently use condom and to have used it during their last sexual intercourse than non-SWs. The sex and numbers of partners also differed between those groups, with a total of $77 \%$ of male SWs reporting either having only male sex partners or having both male and female partners, compared with $8 \%$ among non-SWs. A higher proportion of SWs also reported having had at least 21 male partners in the past 6 months.

\section{Correlates of HIV positivity}

Tables 2 and 3 show univariate and multivariate analyses stratified by sex work among female and male participants.

\section{Women}

Age and recruitment in an urban region were both independently associated with HIV among female participants. While not having completed high school was 


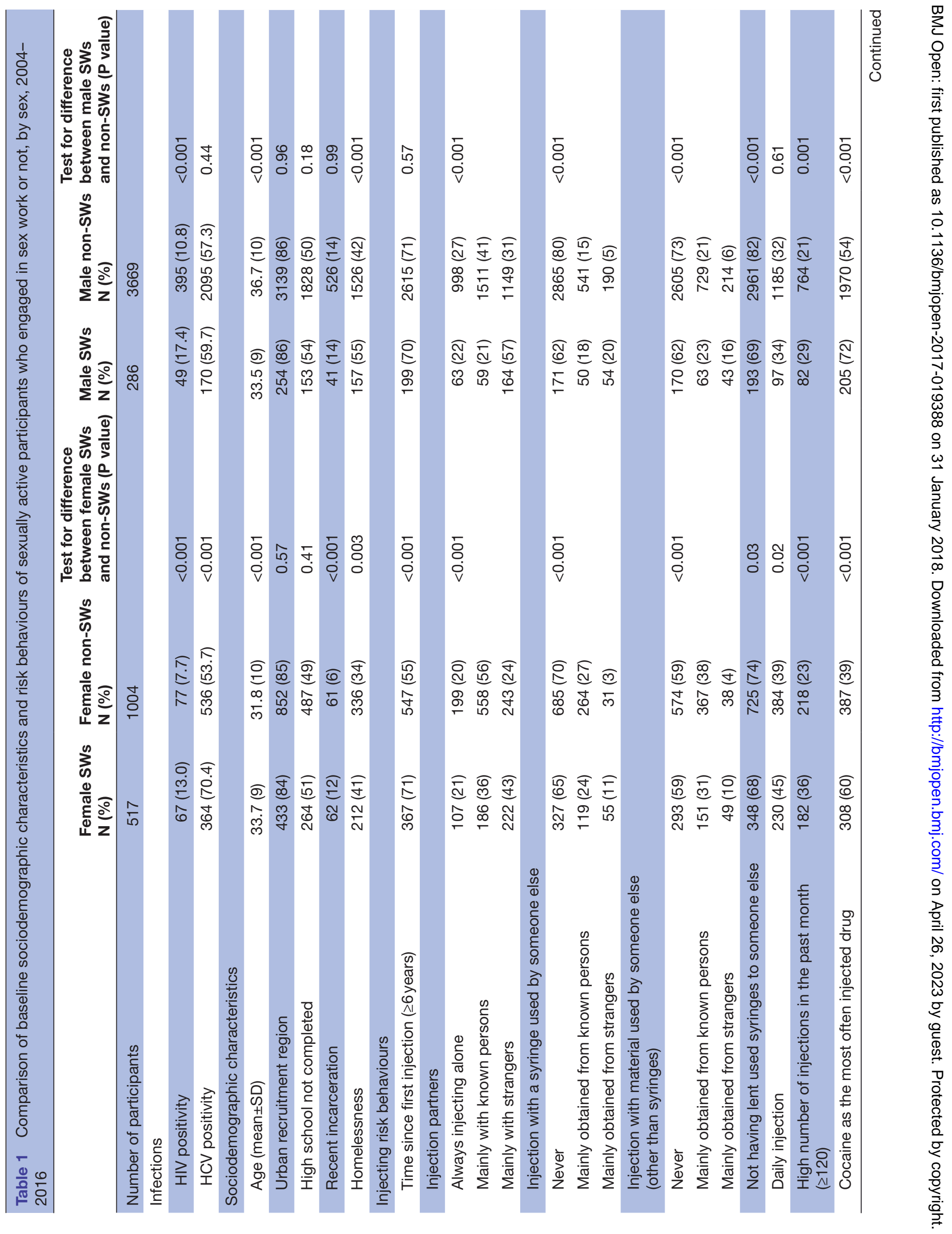




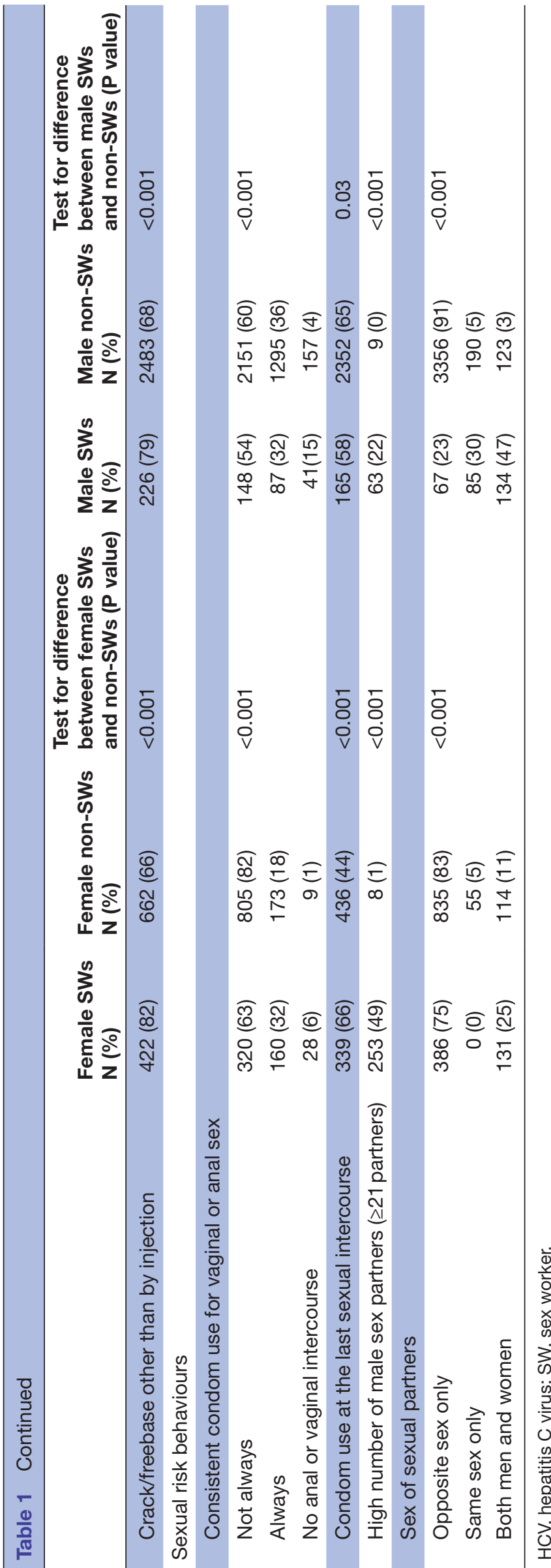

positively associated with HIV positivity among non-SWs only, it almost reached statistical significance among their SWs counterparts. Conversely, female SWs who had been incarcerated recently were more likely to be HIV positive, but this was not the case for non-SWs.

Female SWs who had been injecting since $\geq 6$ years were more likely to be HIV-positive, and injection with syringes obtained mainly from known persons was positively associated with HIV positivity among this group. A similar association was observed among non-SWs, but only concerned injection with syringes obtained mainly from strangers. Cocaine as the most often injected drug was independently associated with HIV positivity in both groups, and female SWs who reported not having lent used syringes to others were 1.89 times more likely of being HIV-positive compared with those who did not report this behaviour.

Furthermore, consistent condom use was found to be associated with HIV positivity among both groups, but having had no anal or vaginal intercourse was positively associated with HIV among SWs only.

\section{Men}

Sociodemographic factors independently associated with HIV positivity among male non-SWs included age and urban recruitment region. Homelessness also appeared as a protective factor among both groups of men.

Men who had been injecting since $\geq 6$ years were more likely to be HIV-positive, irrespectively of whether they were SWs or not. Among SWs, injection with material (other than syringes) mainly obtained from known persons was positively associated with HIV positivity. A similar association was observed among non-SWs, but only concerned injection with syringes mainly obtained from strangers. Both groups of participants who reported not having lent used syringes to others were also more likely of being HIV-positive compared with those who did not report this behaviour.

Having had only male sexual partners was one of the strongest correlates of HIV positivity among all male participants, and the highest prevalence at baseline was found among male SWs who inject drugs and have sex with men $(20.8 \%)$. Additionally, having both female and male sexual partners was associated with HIV among SWs, but not among other male participants. Consistent condom use was also a correlate of HIV positivity among non-SWs, but this association did not remain significant in the multivariate analysis for SWs. Conversely, condom use at the last sexual intercourse was correlated with HIV infection among SWs, but not their non-SWs counterparts.

\section{DISCUSSION}

The objectives of this study were (1) to examine the correlates of HIV positivity among participants who injected and engaged in sex work in the SurvUDI network between 2004 and 2016, after stratification by sex, and (2) to compare these correlates with those of sexually active 


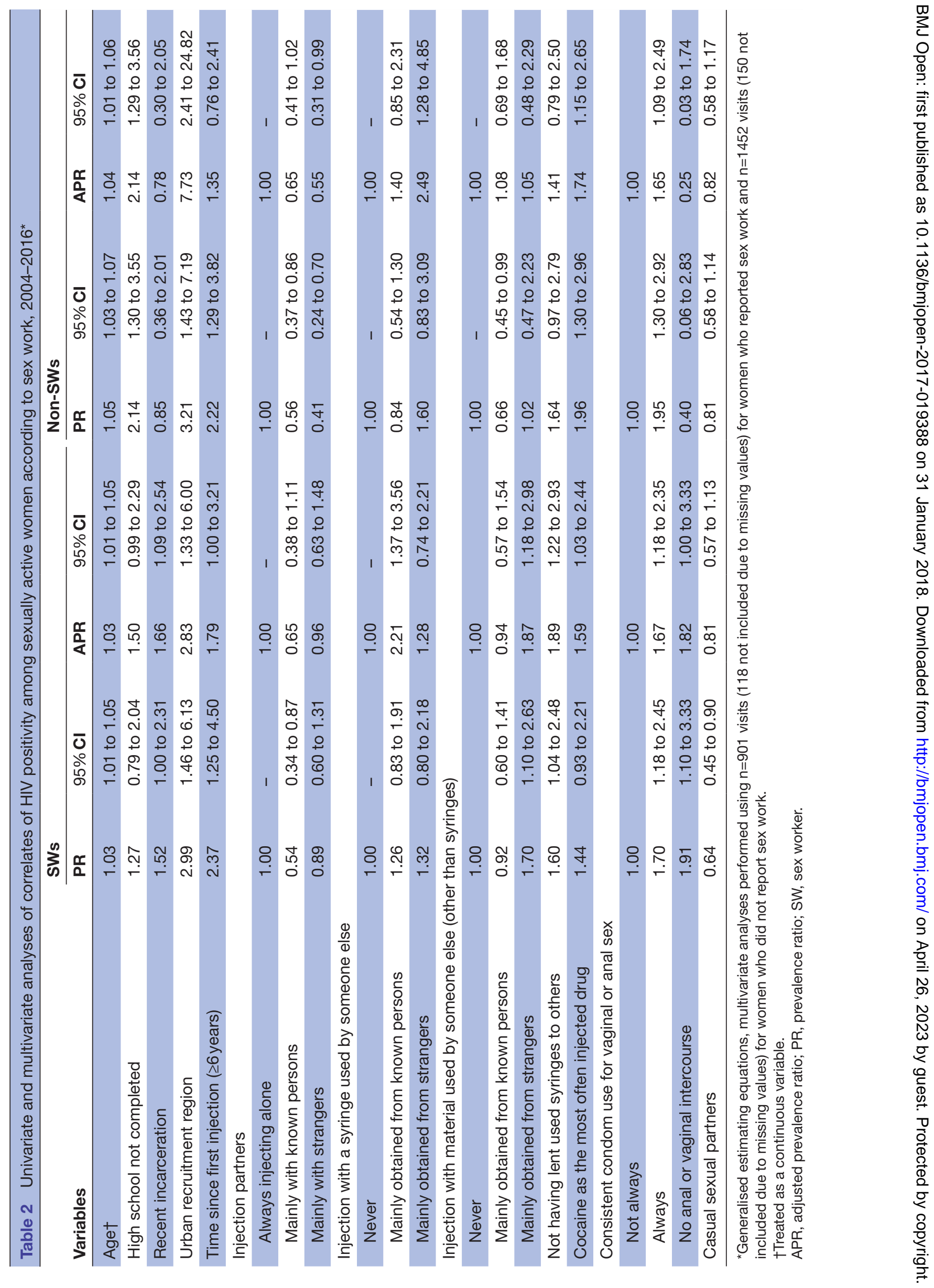




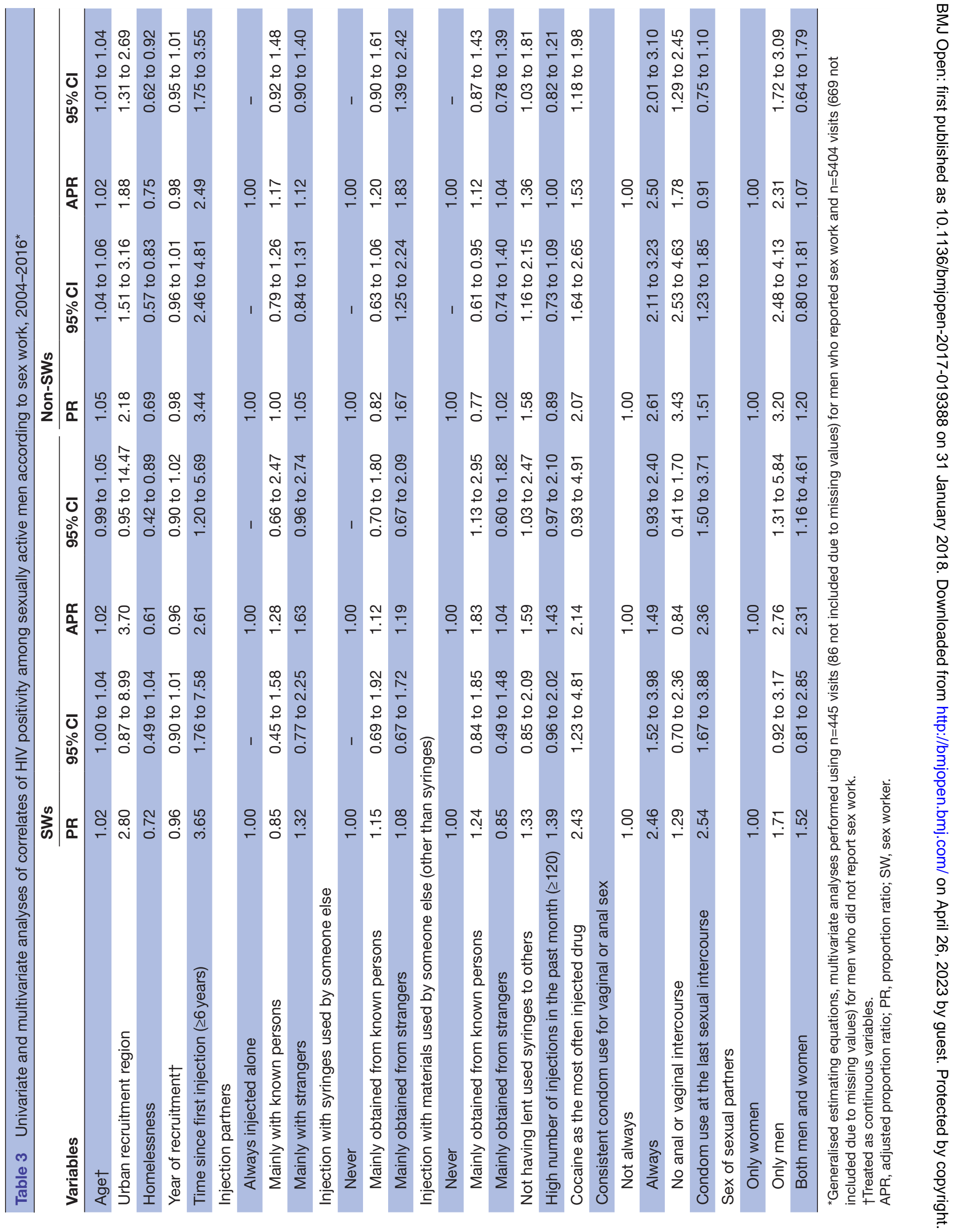


participants who did not engage in sex work. Interpretation of findings should take into account that the large majority (78.7\%) of the HIV-positive participants of the SurvUDI network for the 2004-2016 period were aware of their status.

While we found that a substantial proportion of PWIDs who engage in sex work have experienced homelessness ( $41 \%$ and $55 \%$ among women and men, respectively), homelessness was associated with lower HIV prevalence among men. Considering that a large majority of participants are aware of their status, this result can be interpreted as a higher access to housing subsidies and social programme for people living with HIV, as suggested in a previous study among low-income US urban residents. ${ }^{16}$ Noticeably, women who reported sex work were more likely to have been incarcerated in the past 6 months, possibly due to structural conditions such as the criminalised nature of various aspects of sex work in Canada ${ }^{17}$ and incarceration appeared as a correlate of HIV positivity among this group.

This study also revealed high levels of risk-taking behaviours among SWs and important differences between SW and non-SWs, including higher HIV prevalence among the former. Participants involved in sex work reported multiple injection risk behaviours in higher proportion than non-SWs, confirming findings from previous studies ${ }^{61819}$ and highlighting the need to prioritise, fund and support services to improve prevention services and linkage to care for this specific subgroup. Sexual risk-taking also differed between female and male participants. While a larger proportion of female SWs reported consistent condom use than their non-SWs counterparts, this phenomenon was not observed among male participants.

Despite having examined a variety of sexual risk behaviours for inclusion in our models, our analyses did not identify positive associations between sex-related risk behaviours and HIV among female SWs, suggesting that drug injection behaviours might play a more important role in HIV transmission among this group. By contrast, having only male partners was the strongest correlate of HIV positivity among men, and male PWID-SWs who have sex with men were the most likely to be HIV-positive, with a prevalence of $20.8 \%$ among this group. Almost half of male SWs also reported having both female and male sexual partners, which appeared to double their chances of being HIV-positive, while no association was found in the case of non-SWs. These findings confirm our hypothesis that risky sexual behaviours would be associated with HIV positivity among PWID-SWs who have sex with men. This group has a very high HIV prevalence and should be an important focus for intervention. As suggested elsewhere, those findings indicate that public health practitioners should take the specificities of male PWID-SWs who have sex with men into account when designing prevention programme targeting sexual risk-taking. ${ }^{20}$ A large proportion may not self-identify as homosexual and, as a result, be reached by traditional approaches targeting gay men. ${ }^{20}$
More positively, our results show positive associations between HIV positivity and not lending used syringes as well as consistently using condoms. A plausible explanation for these associations would be reverse causality, that is, that HIV-positive PWIDs might adopt behaviours to protect others. This interpretation is reinforced by the fact that a large majority (78.7\%) of HIV-positive study participants were aware of their HIV-positive status and by previous research suggesting that HIV-positive individuals who are aware of their own serological status tend to adopt protective behaviours. ${ }^{21} 22$

Nonetheless, this was not the case among all participants. A positive association between HIV seropositivity and consistent condom use was also observed among sexually active men, but did not reach significance among those who engage in sex work. HIV-positive men who engage in sex work might be less likely to adopt behaviours to protect their sexual partners than those who do not, possibly due to the dynamic between clients and SWs. It has been reported in previous studies that customers of male SWs rarely ever request that the SW wears a condom and that if a condom was to be worn, clients might cancel the sexual transaction. ${ }^{23} 24$

Another hypothesis is that participants might adopt other strategies to reduce the risk of transmission, such as 'serosorting' (sex with other HIV-positive men) or 'strategic positioning' (adoption of a receptive role during unprotected sex). Those methods, which have been found to be used by HIV-positive men in the USA in a meta-analysis involving 18121 men ${ }^{25}$ can still lead to inadvertent transmission of HIV. ${ }^{26}$ Partners of male PWID-SWs might be at high risk, exposing the need for interventions targeting sexual risk behaviours among this group.

Among the possible limitations of our study, participants might not be representative of all PWIDs since they were mostly recruited through harm reduction programme, thus possibly leading to the over-representation of individuals with more problematic behaviours. Furthermore, the use of self-reported measures may have led to biases because of social desirability, poor recall and intoxication. However, previous studies have shown that PWIDs self-reported behaviours present sufficient validity and reliability. ${ }^{27}{ }^{28}$ These data should be interpreted with caution since it is not possible to verify whether the observed associations between HIV positivity and risk factors are causal, and behaviours may have occurred before or after the time of infection. Finally, these results may not be generalisable to other settings given the importance of the local context in the study of HIV and HCV epidemics among PWIDs.

This study highlights similarities and differences between PWIDs who engage in sex work and those who do not in Eastern Central Canada. Our findings underscore that PWID-SWs are exposed to higher levels of homelessness, incarceration and injecting risk behaviours than their non-SWs counterparts. PWID also have different risk-taking patterns according to implication in sex work, as shown by differences in correlates of HIV positivity. Additionally, risktaking patterns differed between women and men, with a 
larger proportion of injection risk behaviours being associated with HIV among the former and, conversely, a stronger association between sexual behaviours and HIV prevalence being observed among the latter. Public health practitioners should take those specificities into account when designing HIV prevention interventions aimed at PWIDs.

\section{Author affiliations}

${ }^{1}$ Unité sur les Infections Transmissibles Sexuellement et par le Sang, Institut National de Santé Publique du Québec, Montréal, Québec, Canada

2École de santé publique, Université de Montréal, Montréal, Québec, Canada ${ }^{3}$ Direction régionale de Santé Publique - CIUSSS du Centre-Sud-de-l'Île-deMontréal, Montréal, Québec, Canada

${ }^{4}$ Axe Santé des populations et pratiques optimales en santé, Centre de recherche du CHU de Quebec - Université Laval, Québec City, Quebec, Canada

${ }^{5}$ Département de médecine sociale et préventive, Université Laval, Québec City, Québec, Canada

${ }^{6}$ Sérologie, Virologie et biologie moléculaire, Laboratoire de santé publique du Québec, Institut National de Santé Publique du Québec, Sainte-Anne de Bellevue, Québec, Canada

${ }^{7}$ Département des Sciences de la Santé Communautaire, Université de Sherbrooke - Campus de Longueuil, Longueuil, Québec, Canada

Acknowledgements The authors wish to thank the invaluable contribution of interviewers and gratefully acknowledge the contribution of Lise Leblanc and Éric Demers for technical assistance and statistical analyses, respectively. The authors also wish to thank all participants for their excellent collaboration and all members of the SurvUDI Working Group: Nathalie Deshaies, Marie-Michèle Grenier, Geneviève Pouliot-Gagné, Marcel Gauthier, Lynn Potvin, Lynne Leonard, Lina Noël, Nathanaëlle Thériault, Andrée Côté, Marie-Josée Riel, Andrée Perreault and Julie Levesque.

Collaborators Nathalie Deshaies, Marie-Michèle Grenier, Geneviève Pouliot-Gagné, Marcel Gauthier, Lynn Potvin, Lynne Leonard, Lina Noël, Nathanaëlle Thériault, Andrée Côté, Marie-Josée Riel, Andrée Perreault and Julie Levesque

Contributors LC, KB and CB: undertook the analyses . LC drafted the article. ÉR, CM, PL and MA: designed the study. BS: performed and validated laboratory procedures. All authors: provided feedback on drafts, approved the final version and agreed to be accountable for all aspects of the work in ensuring that questions related to the accuracy or integrity of any part of the work are appropriately investigated and resolved.

Funding This study was supported by operating funds from the Public Health Agency of Canada (PHAC) and the Ministère de la santé et des services sociaux du Québec.

Competing interests None declared.

Patient consent Obtained.

Ethics approval Ethics Committee of the Centre de recherche du CHU de Québec.

Provenance and peer review Not commissioned; externally peer reviewed.

Data sharing statement № additional data available.

Open Access This is an Open Access article distributed in accordance with the Creative Commons Attribution Non Commercial (CC BY-NC 4.0) license, which permits others to distribute, remix, adapt, build upon this work non-commercially, and license their derivative works on different terms, provided the original work is properly cited and the use is non-commercial. See: http://creativecommons.org/ licenses/by-nc/4.0/

(C) Article author(s) (or their employer(s) unless otherwise stated in the text of the article) 2018. All rights reserved. No commercial use is permitted unless otherwise expressly granted.

\section{REFERENCES}

1. Kerr T, Shannon K, Ti L, et al. Sex work and HIV incidence among people who inject drugs. AIDS 2016;30:627-34.

2. Merson MH, O'Malley J, Serwadda D, et al. The history and challenge of HIV prevention. Lancet 2008;372:475-88.

3. Hope Ditmore M. When sex work and drug use overlap: considerations for advocacy and practice. London, UK: Harm Reduction International, 2013.
4. Stockman JK, Strathdee SA. HIV among people who use drugs: a global perspective of populations at risk. J Acquir Immune Defic Syndr 2010;55 Suppl 1:S17-S22.

5. Platt L, Rhodes T, Lowndes CM, et al. Impact of gender and sex work on sexual and injecting risk behaviors and their association with HIV positivity among injecting drug users in an HIV epidemic in Togliatti City, Russian Federation. Sex Transm Dis 2005;32:605-12.

6. Croxford S, Platt L, Hope VD, et al. Sex work amongst people who inject drugs in England, Wales and Northern Ireland: findings from a National Survey of Health Harms and Behaviours. Int J Drug Policy 2015;26:429-33.

7. Platt L, Jolley E, Rhodes T, et al. Factors mediating HIV risk among female sex workers in Europe: a systematic review and ecological analysis. BMJ Open 2013;3:605-12.

8. Kuyper LM, Lampinen TM, Li K, et al. Factors associated with sex trade involvement among male participants in a prospective study of injection drug users. Sex Transm Infect 2004;80:531-5.

9. Roy É, Richer I, Morissette C, et al. Temporal changes in risk factors associated with HIV seroconversion among injection drug users in eastern central Canada. AIDS 2011;25:1897-903.

10. Blouin K, Leclerc P, Morissette $C$, et al. Sex work as an emerging risk factor for human immunodeficiency virus seroconversion among people who inject drugs in the SurvUDI Network. Sex Transm Dis 2016;43:648-55.

11. Hankins $\mathrm{C}$, Alary M, Parent R, et al. Continuing HIV transmission among injection drug users in Eastern Central Canada: the SurvUDI Study, 1995 to 2000. J Acquir Immune Defic Syndr 2002;30:514-21.

12. Institut national de santé publique du Québec. Surveillance des maladies infectieuses chez les utilisateurs de drogues par injection - Épidémiologie du VIH de 1995 à 2014 - Épidémiologie du VHC de 2003 à 2014, 2015.

13. Judd A, Parry J, Hickman M, et al. Evaluation of a modified commercial assay in detecting antibody to hepatitis $C$ virus in oral fluids and dried blood spots. J Med Virol 2003;71:49-55.

14. Sullivan Pepe M, Anderson GL. A cautionary note on inference for marginal regression models with longitudinal data and general correlated response data. Commun Stat Simul Comput 1994:23:939-51.

15. Pan W, Louis TA, Connett JE. A note on marginal linear regression with correlated response data. Am Stat 2000;54:191-5.

16. Dickson-Gomez J, McAuliffe T, Convey M, et al. Access to housing subsidies, housing status, drug use and HIV risk among low-income U.S. urban residents. Subst Abuse Treat Prev Policy 2011;6:31.

17. Socías ME, Deering K, Horton M, et al. Social and structural factors shaping high rates of incarceration among sex workers in a Canadian setting. J Urban Health 2015;92:966-79.

18. Roxburgh A, Degenhardt L, Breen C. Drug use and risk behaviours among injecting drug users: a comparison between sex workers and non-sex workers in Sydney, Australia. Harm Reduct J 2005;2:7.

19. Logan TK, Leukefeld C, Farabee D. Sexual and drug use behaviors among women crack users: implications for prevention. AIDS Educ Prev 1998;10:327-40.

20. Bacon O, Lum P, Hahn J, et al. Commercial sex work and risk of HIV infection among young drug-injecting men who have sex with men in San Francisco. Sex Transm Dis 2006;33:228-34.

21. Noor SW, Ross MW, Lai D, et al. Drug and sexual HIV risk behaviours related to knowledge of HIV serostatus among injection drug users in Houston, Texas. Int J STD AIDS 2014;25:89-95.

22. Schlumberger MG, Desenclos JC, Papaevangelou G, et al. Knowledge of HIV serostatus and preventive behaviour among European injecting drug users: second study. European Community Study Group on HIV in Injecting Drug Users. Eur J Epidemiol 1999;15:207-15.

23. Altman D. Men who sell sex: international perspectives on male prostitution and HIVIAIDS. London: Routledge, 1998.

24. Morse EV, Simon PM, Balson PM, et al. Sexual behavior patterns of customers of male street prostitutes. Arch Sex Behav 1992;21:347-57.

25. Crepaz N, Marks G, Liau A, et al. Prevalence of unprotected anal intercourse among HIV-diagnosed MSM in the United States: a meta-analysis. AIDS 2009;23:1617-29.

26. Eaton LA, Kalichman SC, O'Connell DA, et al. A strategy for selecting sexual partners believed to pose little/no risks for HIV: serosorting and its implications for HIV transmission. AIDS Care 2009;21:1279-88.

27. Goldstein MF, Friedman SR, Neaigus A, et al. Self-reports of HIV risk behavior by injecting drug users: are they reliable? Addiction 1995;90:1097-104

28. Darke S. Self-report among injecting drug users: a review. Drug Alcohol Depend 1998;51:253-63. 